\title{
Model reduction design for continuous systems with finite frequency specifications
}

\author{
Miloud Koumir ${ }^{1}$, Abderrahim El-Amrani ${ }^{2}$, Ismail Boumhidi ${ }^{3}$ \\ ${ }^{1}$ LIPI Laboratory, ENS, Sidi Mohamed Ben Abdellah University, Fes, Morocco \\ ${ }^{2,3}$ LISAC Laboratory, FSDM, Sidi Mohamed Ben Abdellah University, Fes, Morocco
}

\begin{tabular}{l}
\hline \hline Article Info \\
\hline Article history: \\
Received Jul 8, 2020 \\
Revised Mar 29, 2021 \\
Accepted Apr 15, 2021 \\
\hline
\end{tabular}

Keywords:

Continuous systems

Finite frequency

Model reduction design

Takagi-sugeno fuzzy scheme

\begin{abstract}
This paper is concerned with the problem of model reduction design for continuous systems in Takagi-Sugeno fuzzy model. Through the defined FF $H_{\infty}$ gain performance, sufficient conditions are derived to design model reduction and to assure the fuzzy error system to be asymptotically stable with a FF $H_{\infty}$ gain performance index. The explicit conditions of fuzzy model reduction are developed by solving linear matrix inequalities. Finally, a numerical example is given to illustrate the effectiveness of the proposed method.
\end{abstract}

This is an open access article under the CC BY-SA license.

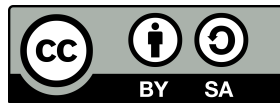

\section{Corresponding Author:}

Abderrahim El-Amrani

LISAC Laboratory

Faculty of Sciences Dhar EL Mehraz (F.S.D.M)

Sidi Mohamed Ben Abdellah University

Fes, Morocco

Email : abderrahim.elamrani@usmba.ac.ma

\section{INTRODUCTION}

In the last few decades, many researchers have investigated model reduction including continuous and discrete settings as these systems have great applications in engineering fields. The problem is to design a low-order model to approach a higher order model given according to some specified criteria. Indeed, many results-based model reduction approach were presented [1]-[10].

In the practical, systems are always more or less disturbed, therefore, model reduction issues for nonlinear systems have been extensively discussed through the T-S fuzzy model approach, see [11]-[16]. Among the most of the existed literature on model reduction problems, the disturbances are considered in the entire frequency (EF) domain, which will bring overdesign in the filtering design. While many practical engineering problems are more suitable to be considered in finite frequency (FF) ranges [17]-[26].

The main objective of this paper is to design a model reduction for continuous T-S fuzzy systems with disturbance in FF domain. Through the defined FF $H_{\infty}$ gain performance, sufficient conditions are derived to design model reduction and to assure the fuzzy error system to be asymptotically stable with a FF $H_{\infty}$ gain performance index. The explicit conditions of fuzzy FF are developed by solving linear matrix inequalities. A systematic model reduction design scheme is proposed, which could reduce the conservatism of the results compared to the one considered in EF domain. Finally, a simulation example demonstrates the usefulness of the proposed method.

Notations: The notation $A>0(A \leq 0)$ means that $A$ is positive definite (positive semi-definite). $A^{-1}, A^{T}, A^{*}$ denote the inverse, the transpose and the complex conjugate transpose of matrix $A$, respectively. Symbol ${ }^{\prime} *^{\prime}$ represents the term originated by conjugate symmetry in a matrix. 


\section{PROBLEM FORMULATION}

The plant under consideration is a continuous T-S fuzzy system described by its i-th rule as follows:

Plant Rule $i$ : IF $\sigma_{1}(t)$ is $T_{1}^{i}, \ldots$ and $\sigma_{p}(t)$ is $T_{p}^{i}$ THEN,

$$
\begin{aligned}
\dot{x}(t) & =A_{i} x(t)+B_{i} u(t) \\
y(t) & =C_{i} x(t)
\end{aligned}
$$

where $x(t) \in \mathbb{R}^{n}$ is the state vector; $y(t) \in \mathbb{R}^{p}$ is the measured output vector; $u(t) \in \mathbb{R}^{m}$ is the external noise signal of the following frequency sets,

$$
\Delta \triangleq\left\{\begin{array}{lcc}
\omega \in \mathbb{R}|| \omega \mid \leq \bar{\omega}_{l}, & \bar{\omega}_{l} \geq 0, & (L F) \\
\omega \in \mathbb{R} \mid \bar{\omega}_{1} \leq \omega \leq \bar{\omega}_{2} & \bar{\omega}_{1}, \bar{\omega}_{2} \in[0,+\infty], & (M F) \\
\omega \in \mathbb{R}|| \omega \mid \geq \bar{\omega}_{h}, & \bar{\omega}_{h} \geq 0, & (H F)
\end{array}\right.
$$

where LF, MF and HF stand for low-, middle-, and high- frequency ranges, respectively.

Via using inference product, singleton fuzzifer and center-average defuzzifer, nonlinear system (1) can be described by:

$$
\begin{aligned}
& \dot{x}(t)=A(\rho) x(t)+B(\rho) u(t) \\
& y(t)=C(\rho) x(t)
\end{aligned}
$$

where

$$
\begin{aligned}
& A(h)=\sum_{i=1}^{r} h_{i} A_{i} ; \quad B(h)=\sum_{i=1}^{r} h_{i} B_{i} ; \\
& C(h)=\sum_{i=1}^{r} h_{i} C_{i}
\end{aligned}
$$

and

$$
\begin{gathered}
h_{i}(\sigma(t))=\frac{\prod_{j=1}^{p} \mu_{i j}\left(\sigma_{j}(t)\right)}{\sum_{i=1}^{r} \prod_{j=1}^{p} \mu_{i j}\left(\sigma_{j}(t)\right)} \\
\rho_{i} \geq 0, \quad i=1, \ldots, r, \quad \sum_{i=1}^{r} \rho_{i}=1 .
\end{gathered}
$$

with $h:=\left(h_{1}, h_{2}, \ldots, h_{r}\right) \in \delta$.

In this paper, we are interested in approximating the T-S fuzy system (4) by a stable $\hat{n}$ th-order $(\hat{n}<n)$ reduced-order T-S model.

Plant Rule $i$ : $\operatorname{IF} \sigma_{1}(t)$ is $N_{1}^{i}, \ldots$ and $\sigma_{p}(t)$ is $N_{p}^{i}$ THEN

$$
\begin{aligned}
& \hat{\dot{x}}(t)=\hat{A}_{i} \hat{x}(t)+\hat{B}_{i} u(t) \\
& \hat{z}(t)=\hat{C}_{i} \hat{x}(t)
\end{aligned}
$$

where $\hat{x}(t) \in \mathbb{R}^{\hat{n}}(\hat{n}<n)$ is the state of the reduced-order model, $\hat{y}(t) \in \mathbb{R}^{p}$ is the output of the reduced-order model.

Then, the fuzzy reduced-order model as (8),

$$
\begin{aligned}
\left(\Sigma_{r}\right): \hat{\dot{x}}(t) & =\hat{A}(\rho) \hat{x}(t)+\hat{B}(\rho) u(t) \\
\hat{y}(t) & =\hat{C}(\rho) \hat{x}(t)
\end{aligned}
$$


where

$$
\begin{aligned}
\hat{A}(\rho) & =\sum_{i=1}^{r} \rho_{i}(t) \hat{A}_{i}, \quad \hat{B}(\rho)=\sum_{i=1}^{r} \rho_{i}(t) \hat{B}_{i} \\
\hat{C}(\rho) & =\sum_{i=1}^{r} \rho_{i}(t) \hat{C}_{i}
\end{aligned}
$$

Then, we have the error model:

$$
\begin{aligned}
\left(\Sigma_{e}\right): \dot{\mathcal{X}}(t) & =\bar{A}(\rho, \rho) \mathcal{X}(t)+\bar{B}(\rho, \rho) u(t) \\
\mathcal{E}(t) & =\bar{C}(\rho, \rho) \mathcal{X}(t)+\bar{D}(\rho, \rho) u(t)
\end{aligned}
$$

where

$$
\begin{aligned}
\bar{A}(\rho, \rho) & =\left[\begin{array}{cc}
A(\rho) & 0 \\
0 & \hat{A}(\rho)
\end{array}\right] ; \bar{B}(\rho, \rho)=\left[\begin{array}{c}
B(\rho) \\
\hat{B}(\rho)
\end{array}\right] \\
\bar{C}(\rho, \rho) & =\left[\begin{array}{cc}
C(\rho) & -\hat{C}(\rho)
\end{array}\right] \\
\mathcal{X}(t) & =\left[\begin{array}{l}
x(t) \\
\hat{x}(t)
\end{array}\right] ; \mathcal{E}(t)=y(t)-\hat{y}(t)
\end{aligned}
$$

Next, given a scalar $\gamma$ and a rectangular FF domain, the error system $\left(\Sigma_{e}\right)$ is said to have a FF $H_{\infty}$ performance if it satisfies the following inequality holds,

$$
\int_{\omega \in \triangle} E^{T}(\omega) E(\omega) d \omega \leq \gamma^{2} \int_{\omega \in \triangle} U^{T}(\omega) U(\omega) d \omega
$$

where

$$
\triangle \triangleq\left\{w \in \mathbb{R}:|\omega| \leq \omega_{l} ; \omega_{l} \in[0,+\infty]\right\}
$$

The following inequalities hold [27]:

$$
\begin{aligned}
& \Theta_{i i}<0 ; \quad 1 \leq i \leq r \\
& \frac{1}{r-1} \Theta_{i i}+\frac{1}{2}\left[\Theta_{i j}+\Theta_{j i}\right]<0 ; \quad 1 \leq i \neq j \leq r
\end{aligned}
$$

where

$$
\sum_{i=1}^{r} \sum_{j=1}^{r} \rho_{i}(t) \rho_{j}(t) \Theta_{i j}<0
$$

Let $\triangle \in \mathbb{R}^{n}, \mathcal{J} \in \mathbb{R}^{n \times n}, \mathcal{X} \in \mathbb{R}^{m \times n}, \operatorname{rank}(\mathcal{X})=r<n$ and $\mathcal{X}^{\perp} \in \mathbb{X}^{n \times(n-r)}$ such that $\mathcal{X} \mathcal{X}^{\perp}=0$ [28], so that the following conditions are equivalent:

$$
\begin{aligned}
& -\triangle^{*} \mathcal{J} \triangle<0, \forall \triangle \neq 0: \mathcal{X} \triangle=0 \\
& -\mathcal{X}^{\perp *} \mathcal{J} \mathcal{X}^{\perp}<0 \\
& -\exists \mathcal{T} \in \mathbb{R}^{n \times m}: \mathcal{J}+\mathcal{T} \mathcal{X}+\mathcal{X}^{*} \mathcal{T}^{*}<0
\end{aligned}
$$

Error system (10) is stable and the FF $H_{\infty}$ performance (12) is satisfied if there exist $\mathcal{P}=\mathcal{P}^{T}, \mathcal{Q}=\mathcal{Q}^{T}>0$ such that [29].

$$
\left[\begin{array}{cc}
\bar{A}(\rho, \rho) & \bar{B}(\rho, \rho) \\
I & 0
\end{array}\right]^{T}\left[\begin{array}{cc}
-\mathcal{Q} & \mathcal{P} \\
\mathcal{P} & \omega_{l}^{2} \mathcal{Q}
\end{array}\right]\left[\begin{array}{cc}
\bar{A}(\rho, \rho) & \bar{B}(\rho, \rho) \\
I & 0
\end{array}\right]+\left[\begin{array}{cc}
\bar{C}^{T}(\rho, \rho) \bar{C}(\rho, \rho) & 0 \\
0 & -\gamma^{2} I
\end{array}\right]<0
$$




\section{FF $H_{\infty}$ PERFORMANCE DESIGN}

Error model (8) is stable, if $\mathcal{P} \in \mathbb{H}_{n}, 0<\mathcal{Q} \in \mathbb{H}_{n}, 0<\mathcal{W} \in \mathbb{H}_{n}, \mathcal{F}, \mathcal{G}, \mathcal{L} \mathcal{H}$ such that

$\Upsilon=\left[\begin{array}{cc}-\mathcal{F}-\mathcal{F}^{T} & \mathcal{W}+\mathcal{F} \bar{A}(\rho, \rho)-\mathcal{G}^{T} \\ * & \operatorname{He}\{\mathcal{G} \bar{A}(\rho, \rho)\}\end{array}\right]<0$

$\Omega=\left[\begin{array}{cccc}-\mathcal{Q}-H e[\mathcal{F})] & \mathcal{P}-\mathcal{G}^{T}+\mathcal{F} \bar{A}(\rho, \rho) & \mathcal{F} \bar{B}(\rho, \rho)-\mathcal{L}^{T}(\rho, \rho) & -\mathcal{H}^{T} \\ * & \omega_{l}^{2} \mathcal{Q}+H e[\mathcal{G} \bar{A}(\rho, \rho)] & \mathcal{G} \bar{B}(\rho, \rho)+\bar{A}^{T}(\rho, \rho) \mathcal{L}^{T}(\rho, \rho) & \left.\bar{C}^{T}(\rho, \rho)+\bar{A}^{T} \rho, \rho\right) \mathcal{H}^{T} \\ * & * & -\gamma^{2} I+H e[\mathcal{L} \bar{B}(\rho, \rho)] & \left.\bar{B}^{T} \rho, \rho\right) \mathcal{H}^{T} \\ * & * & * & -I\end{array}\right]<0$

First. Define:

$$
\begin{aligned}
& {\left[\begin{array}{c}
\bar{A}(\rho, \rho) \\
I
\end{array}\right]^{T}\left[\begin{array}{cc}
0 & \mathcal{W} \\
\mathcal{W} & 0
\end{array}\right]\left[\begin{array}{c}
\bar{A}(\rho, \rho) \\
I
\end{array}\right]<0}
\end{aligned}
$$

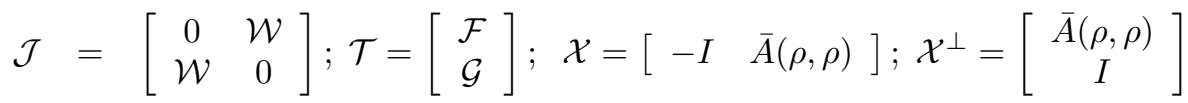

then

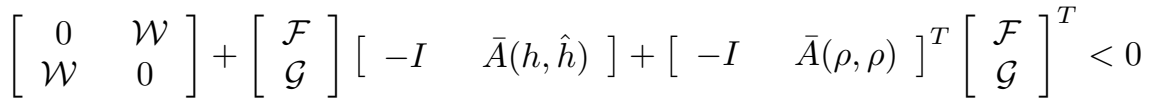

which is nothing but (17).

On the other hand, Let:

$$
\begin{aligned}
\mathcal{J} & =\left[\begin{array}{cccc}
-\mathcal{Q} & \mathcal{P} & 0 & 0 \\
\mathcal{P} & \omega_{l}^{2} \mathcal{Q} & 0 & \bar{C}^{T}(\rho, \rho) \\
0 & 0 & -\gamma^{2} I & 0 \\
0 & \bar{C}(\rho, \rho) & 0 & -I
\end{array}\right] ; \mathcal{T}=\left[\begin{array}{llll}
\mathcal{F}^{T} & \mathcal{G}^{T} & \mathcal{L}^{T} & \mathcal{H}^{T}
\end{array}\right]^{T} \\
\mathcal{X} & =\left[\begin{array}{llll}
-I & \bar{A}(\rho, \rho) & \bar{B}(\rho, \rho) & 0
\end{array}\right]
\end{aligned}
$$

then, we obtain (16). Error system in (8) is stable with an $H_{\infty}$ performance bound $\gamma$, if there exist $\mathcal{P}=\left[\begin{array}{cc}\mathcal{P}_{1} & \mathcal{P}_{2} \\ * & \mathcal{P}_{3}\end{array}\right], Q=\left[\begin{array}{cc}\mathcal{Q}_{1} & \mathcal{Q}_{2} \\ * & \mathcal{Q}_{3}\end{array}\right]>0, \mathcal{W}=\left[\begin{array}{cc}\mathcal{W}_{1} & \mathcal{W}_{2} \\ * & \mathcal{W}_{3}\end{array}\right]>0, \breve{\mathcal{A}}_{i}, \breve{\mathcal{B}}_{i}, \breve{\mathcal{C}}_{i}, \mathcal{F}_{1}, \mathcal{F}_{2}, \mathcal{G}_{1}, \mathcal{G}_{2}, \mathcal{H}_{1}$, $E=\left[\begin{array}{ll}I & 0\end{array}\right]^{T}$ and $\mathcal{V}$, such that

$$
\begin{gathered}
\tilde{\Omega}_{i i}<0 ; \quad \tilde{\Upsilon}_{i i}<0 ; \quad 1 \leq i \leq r \\
\frac{1}{r-1} \tilde{\Omega}_{i i}+\frac{1}{2}\left[\tilde{\Omega}_{i j}+\tilde{\Omega}_{j i}\right]<0 ; \quad 1 \leq i \neq j \leq r \\
\frac{1}{r-1} \tilde{\Upsilon}_{i i}+\frac{1}{2}\left[\tilde{\Upsilon}_{i j}+\tilde{\Upsilon}_{j i}\right]<0 ; \quad 1 \leq i \neq j \leq r
\end{gathered}
$$




$$
\begin{aligned}
& \tilde{\Omega}=\left[\begin{array}{cccccc}
\tilde{\Omega}_{11} & \tilde{\Omega}_{12} & \mathcal{P}_{1}-\mathcal{G}_{1}^{T}+\mathcal{F}_{1} A_{j} & \mathcal{P}_{2}-\mathcal{G}_{2}^{T}+E \breve{\mathcal{A}}_{i} & \mathcal{F}_{1} B_{j}+E \breve{\mathcal{B}}_{i}-\mathcal{L}_{1}^{T} & -\mathcal{H}_{1}^{T} \\
* & \tilde{\Omega}_{22} & \mathcal{P}_{2}-\mathcal{V}^{T} E^{T}+\mathcal{F}_{2} A_{j} & \mathcal{P}_{3}-\mathcal{V}^{T}+\breve{\mathcal{A}}_{i} & \mathcal{G}_{2} B_{j}+\breve{\mathcal{A}}_{i} & A_{j}^{T} \mathcal{H}_{1}^{T} \\
* & * & \omega_{l}^{2} \mathcal{Q}_{1}+\mathcal{G}_{1} A_{j}+A_{j}^{T} \mathcal{G}_{1}^{T} & \omega_{l}^{2} \mathcal{Q}_{2}+A_{j}^{T} \mathcal{G}_{2}^{T}+E \breve{\mathcal{A}}_{i} & \mathcal{G}_{1} B_{j}+E \breve{\mathcal{B}}_{i}+A_{j}^{T} \mathcal{L}_{1}^{T} & C_{j}^{T} \\
* & * & * & \breve{\mathcal{C}}_{j}^{T} \\
* & * & * & \omega_{l}^{2} \mathcal{Q}_{3}+\breve{\mathcal{A}}_{i}+E \breve{\mathcal{A}}_{i}^{T} & \mathcal{G}_{2} B_{j}+\breve{\mathcal{B}}_{i} & -\gamma^{2} \\
* & * & * & * & -\gamma^{2} I+H\left[\mathcal{L}_{1} B_{j}\right] & B_{j}^{T} \mathcal{H}_{1}^{T} \\
& * & * & * & -I
\end{array}\right] \\
& \tilde{\Upsilon}=\left[\begin{array}{cccc}
-\mathcal{F}_{1}-\mathcal{F}_{1}^{T} & -E \mathcal{V}-\mathcal{F}_{2}^{T} & \mathcal{P}_{1}-\mathcal{G}_{1}^{T}+\mathcal{F}_{1} A_{j} & \mathcal{P}_{2}-\mathcal{G}_{2}^{T}+E \breve{\mathcal{A}}_{i} \\
* & -\mathcal{V}-\mathcal{V}^{T} & \mathcal{W}_{2}+\mathcal{F}_{2} A_{j}-\mathcal{V}^{T} E^{T} & \mathcal{W}_{3}+\breve{\mathcal{A}}_{i}-\mathcal{V}^{T} \\
* & * & \mathcal{G}_{1} A_{j}+A_{j}^{T} \mathcal{G}_{1}^{T} & E \breve{\mathcal{A}}_{j}+A_{j}^{T} \mathcal{G}_{2}^{T} \\
* & * & * & H e\left[\breve{\mathcal{A}}_{i}\right]
\end{array}\right] \\
& \bar{\Omega}_{11}=\mathcal{Q}_{1}-\mathcal{F}_{1}-\mathcal{F}_{1}^{T} ; \bar{\Omega}_{12}=\mathcal{Q}_{2}-E \mathcal{V}-\mathcal{F}_{2}^{T} ; \bar{\Omega}_{22}=\mathcal{Q}_{3}-\mathcal{V}-\mathcal{V}^{T}
\end{aligned}
$$

Built on Th. 3., we pick of parameters $\mathcal{F}, \mathcal{G}, \mathcal{L}, \mathcal{H}$ :

$$
\begin{aligned}
\mathcal{F} & =\left[\begin{array}{cc}
\mathcal{F}_{1} & E \mathcal{V} \\
\mathcal{F}_{2} & \mathcal{V}
\end{array}\right] ; \mathcal{G}=\left[\begin{array}{cc}
\mathcal{G}_{1} & E \mathcal{V} \\
\mathcal{G}_{2} & \mathcal{V}
\end{array}\right] ; \\
\mathcal{L} & =\left[\begin{array}{ll}
\mathcal{L}_{1} & 0
\end{array}\right] ; \mathcal{H}=\left[\begin{array}{ll}
\mathcal{H}_{1} & 0
\end{array}\right]
\end{aligned}
$$

and

$$
\breve{\mathcal{A}}(\rho)=\mathcal{V} \hat{A}(\rho) ; \breve{\mathcal{B}}(\rho)=\mathcal{V} \hat{B}(\rho)
$$

Finally, by applying Lemma 2, we have Th. 3 .

Moreover, under the above conditions, we can obtain a state-space realization of model reduction (8) with the following parameters as (27).

$$
\breve{A}_{i}=\mathcal{V}^{-1} \hat{A}_{i} ; \quad \breve{B}_{i}=\mathcal{V}^{-1} \hat{B}_{i} ; ; \quad \breve{C}_{i}=\hat{C}_{i}
$$

\section{NUMERICAL EXAMPLE}

Consider tunnel diode circuit shown in Figure 1 with two rules [30]:

Plant Rule 1: IF $x_{1}(t)$ is $M_{1}\left(x_{1}(t)\right)$ THEN

$$
\begin{aligned}
\dot{x}(t) & =A_{1} x(t)+B_{1} u(t) \\
y(t) & =C_{1} x(t)
\end{aligned}
$$

Plant Rule 2: IF $x_{1}(t)$ is $M_{2}\left(x_{1}(t)\right)$ THEN

$$
\begin{aligned}
\dot{x}(t) & =A_{2} x(t)+B_{2} u(t) \\
y(t) & =C_{2} x(t)
\end{aligned}
$$

where

$$
\begin{aligned}
A_{1}= & {\left[\begin{array}{cccc}
-0.2 & 100 & 0 & 0 \\
-10 & -66.6667 & 3.3333 & -66.6667 \\
0 & -33.3333 & -1.6667 & -16.6667 \\
0 & -33.3333 & -1.6667 & -33.3333
\end{array}\right] ; \quad B_{1}=B_{2}=\left[\begin{array}{c}
0 \\
0 \\
-1.6667 \\
-3.3333
\end{array}\right] } \\
A_{2}= & {\left[\begin{array}{cccc}
-9.2 & 100 & 0 & 0 \\
-10 & -66.6667 & 3.3333 & -66.6667 \\
0 & -33.3333 & -1.6667 & -16.6667 \\
0 & -33.3333 & -1.6667 & -33.3333
\end{array}\right] ; \quad C_{1}=C_{2}=\left[\begin{array}{llll}
1 & 0 & 0 & 0
\end{array}\right] }
\end{aligned}
$$


The membership functions:

$$
M_{1}\left(x_{1}(t)\right)=1-\frac{x_{1}^{2}(t)}{9} ; M_{2}\left(x_{1}(t)\right)=1-M_{1}\left(x_{1}(t)\right)
$$

when $x_{1}(t)$ is almost \pm 3 and 0 .

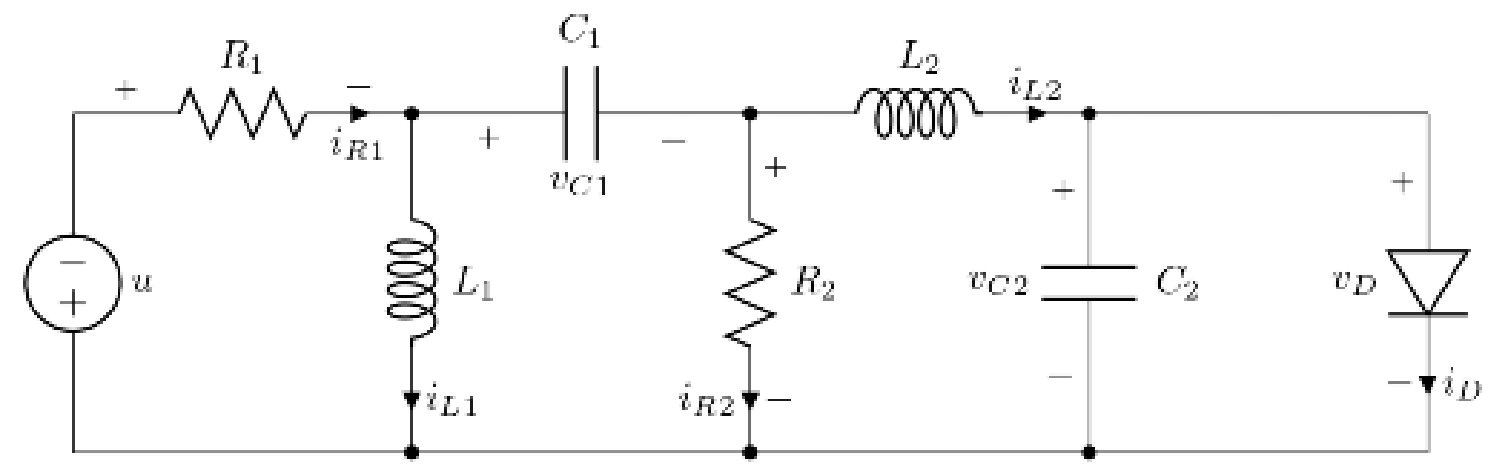

Figure 1. A tunnel diode circuit

As an example, let the disturbance input be the following form (32).

$$
u(t)=0.1 \sin (2 t), \quad \text { i.e., } .|\omega| \leq 2 \mathrm{rad} / \mathrm{s} .
$$

By Theorem 3, the reduced-order models are given by (33),

$$
\begin{aligned}
& {\left[\begin{array}{c|c}
\hat{A}_{1} & \hat{B}_{1} \\
\hline \hat{C}_{1} & -
\end{array}\right]=\left[\begin{array}{cc|c}
-11.1147 & 55.2147 & -8.7325 \\
-1.2574 & -4.1258 & -1.0245 \\
\hline-0.2487 & 0.4175 & -
\end{array}\right]} \\
& {\left[\begin{array}{c|c}
\hat{A}_{2} & \hat{B}_{2} \\
\hline \hat{C}_{2} & -
\end{array}\right]=\left[\begin{array}{ccc}
-10.4785 & 56.0147 & -9.0147 \\
-1.2104 & -4.3301 & -0.9782 \\
\hline-0.2501 & 0.3305 & -
\end{array}\right]}
\end{aligned}
$$

We propose in Table 1 shows the values of $\gamma$ obtained in different frequency ranges. We can see from Table 1 shows the values of $\gamma$ obtained with the approaches existing in [5], [22], [23] and Theorem 3. We can see that the proposed method provides better results than the existing ones.

Table 1. $H_{\infty}$ performance bounds $\gamma$ by different domains

\begin{tabular}{cccr}
\hline Frequency & Methods & $\gamma_{\min }$ & Max error \\
\hline $0 \leq \omega \leq \infty$ & {$[5]$} & 1.1457 & - \\
$|\omega| \leq 2$ & {$[22]$} & 0.3587 & 0.3245 \\
$|\omega| \leq 2$ & {$[23]$} & 0.2324 & 0.2325 \\
$|\omega| \leq 2$ & Th 3. & 0.1279 & 0.0332 \\
\hline
\end{tabular}

Next, let:

$$
\mu(t)=\sqrt{\sum_{t=0}^{\infty} \mathcal{E}^{T}(t) \mathcal{E}(t) / \sum_{t=0}^{\infty} u^{T}(t) u(t)}
$$

Figures 2(a) and (b) present the estimation error $\mathcal{E}(t)$ for different methods and evolution of ratio $\mu(t)$ in (34). From Figure 2(a), the asymptotic stability of the error system can be clearly observed, while under the zero boundary conditions and the disturbance input (32). From Figure 2(b), the ratio tends to a constant value 0.1234 in $|\omega| \leq 2$ domains. 


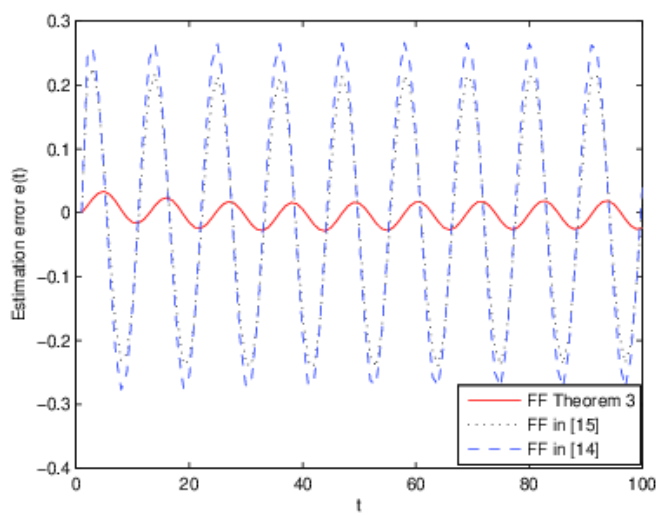

(a)

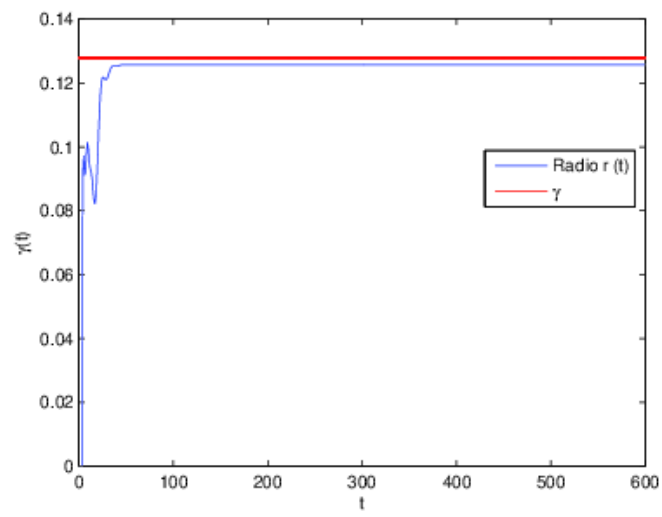

(b)

Figure 2. Trajectories of $\mathcal{E}(t)$ and $\mu(t)$ for $|\omega| \leq 2$ range; (a) Estimation error $\mathcal{E}(t)$ from different methods,

(b) Estimation $\mu(t)$

\section{CONCLUSION}

This paper has concerned with the problem of the model reduction design for continuous T-S fuzzy systems with FF disturbances. Assuming the disturbances is dominated in a known FF domain. Through applying a more general linearization procedure, systematic methods have been proposed for model reduction design, which guarantees the asymptotic stability and the FF $H_{\infty}$ gain performance of the error system. A simulation example has been given to illustrate the effectiveness of the proposed method.

\section{REFERENCES}

[1] A. Antoulas, "Approximation of large-scale dynamical systems," SIAM, Philadelphia, 2005.

[2] K. Glover, "All optimal Hankel-norm approximations of linear multivariable systems and their 11 error bounds," Int. J. Control, vol. 39, no. 6, pp. 1115-1195, 1984.

[3] G. J. Lastman, "Reduced-order aggregated models for bilinear time invariant dynamical systems," IEEE Trans. Automat. Contr., vol. 29, pp. 359-361, 1984.

[4] B. Moore, "Principal component analysis in linear systems: controllability, observability, and model reduction," IEEE Trans. Automat. Contr., vol. 26, no. 1, pp. 17-32, 1981.

[5] X. Su, L. Wu, P. Shi, and Y.-D. Song, " $H_{\infty}$ model reduction of Takagi-Sugeno fuzzy stochastic systems," IEEE Transactions on Systems, Man, and Cybernetics-Part B: Cybernetics, vol. 42, no. 6, pp. 1574-1585, 2012, doi: 10.1109/tsmcb.2012.2195723.

[6] L. Wu, X. Su, P. Shi, and J. Qiu, "Model approximation for discrete-time state-delay systems in the T-S fuzzy framework," IEEE Trans. Fuzzy Syst., vol. 19, no. 2, pp. 366-378, Apr. 2011.

[7] A. El-Amrani, A. El Hajjaji, B. Boukili, I. Boumhidi and A. Hmamed, "Robust over finite frequency ranges $H_{\infty}$ model reduction for uncertain 2D continuous systems," International Journal of System of Systems Engineering, vol. 10, no. 2, pp. 108-127, 2020.

[8] A. El-Amrani, B. Boukili, A. El Hajjaji, A. Hmamed and I. Boumhidi, "Robust finite frequency $H_{\infty}$ model reduction for uncertain 2D continuous systems," IEEE 58th Conference on Decision and Control (CDC), 2019, pp. 5629-5634.

[9] A. Bonito, A. Cohen, R. DeVore, D. Guignard, P. Jantsch and G. Petrova, "Nonlinear methods for model reduction," ESAIM: Mathematical Modelling and Numerical Analysis, vol. 55, no. 2, pp. 507-531, 2021.

[10] A. El-Amrani, A. E. Hajjaji, I. Boumhidi, A. Hmamed, and A. Aitouche, "Finite Frequency Approach for $H_{\infty}$ model reduction of 2D continuous systems," In 2019 27th Mediterranean Conference on Control and Automation (MED), 2019, pp. 177-182.

[11] T. Takagi and M. Sugeno, "Fuzzy identification of systems and its applications to modeling and control," IEEE Trans. Syst., Man, Cybern., vol. SMC-15, no. 1, pp. 116-132, Jan./Feb. 1985.

[12] H. Zhang, Y. Shi and A. S. Mehr, "On $H_{\infty}$ Filtering for Discrete-Time Takagi-Sugeno Fuzzy Systems," IEEE Transactions on Fuzzy Systems, vol. 20, no 2, pp. 396-401, 2012.

[13] A. Benzaouia, A. Hmamed and A. EL Hajjaji, "Stabilization of controlled positive discrete-time T-S fuzzy systems by state feedback control," International Journal of Adaptive Control and Signal Processing, vol. 24, pp. 1113-1121, 2010, doi: 10.1002/acs.1185. 
[14] H. Gao, Y. Zhao, J. Lam, and K. Chen, " $H_{\infty}$ fuzzy filtering of nonlinear systems with intermittent measurements," IEEE Transactions on Fuzzy Systems, vol. 17, no. 2, pp. 291-300, 2009.

[15] A. El-Amrani, A. El Hajjaji, A. Hmamed, and I. Boumhidi, "Finite Frequency Filter Design for T-S Fuzzy Continuous Systems," IEEE International Conference on Fuzzy Systems (FUZZ-IEEE), 2018, pp. 1-7.

[16] A. El-Amrani, I. Boumhidi, B. Boukili and A. Hmamed, "A finite frequency range approach to $H_{\infty}$ filtering for TS fuzzy systems," Procedia computer science, vol. 148, pp. 485-494, 2019.

[17] D. Wang, and A. Zilouchian, "Model reduction of discrete linear systems via frequency-domain balanced structure," IEEE Trans. Circuits Syst. I, vol. 47, no. 6, pp. 830-837, 2000.

[18] X. Du and G.-H. Yang, " $H_{\infty}$ model reduction of linear continuous time systems over finie frequency interval," IET Control Theory and Applications, vol. 4, no. 3, pp. 499-508, 2010.

[19] X. Li, S. Yin, H. Gao, "Passivity-preserving model reduction with finite frequency $H_{\infty}$ approximation performance," Automatica, vol. 50, no. 9, pp. 2294-2303, 2014.

[20] D. W. Ding, X. Xie, X. Du, and X. J. Li, "Finite-frequency model reduction of discrete-time T-S fuzzy state-delay systems," Neurocomputing, vol. 203, pp. 121-128, 2016.

[21] S. Aboulem, A. El-Amrani and I. Boumhidi, "Finite frequency $H_{\infty}$ control for wind turbine systems in TS form," International Journal of Power Electronics and Drive Systems (IJPEDS), vol. 11, no. 3, pp. 1-10, 2020, doi: 10.11591/ijpeds.v11.i3.pp1313-1322.

[22] D. -W. Ding, X. -J. Li, X. Du, and X. Xie, "Finite-Frequency Model Reduction of Takagi-Sugeno Fuzzy Systems," IEEE Transactions on Fuzzy Systems, vol. 24, no. 6, pp. 1464-1474, 2016.

[23] A. El-Amrani, B. Boukili, A. El Hajjaji and A. Hmamed, " $H_{\infty}$ model reduction for T-S fuzzy systems over finite frequency ranges," Optimal Control Applications and Methods, vol. 39, no 4, pp. 1479-1496, 2018.

[24] A. El-Amrani, B. Boukili, A. Hmamed, and I. Boumhidi, " $H_{\infty}$ model reduction for TS fuzzy systems with finite frequency specifications," In 2017 Intelligent Systems and Computer Vision (ISCV), pp. 1-7, 2017.

[25] A. El-Amrani, A. E. Hajjaji, I. Boumhidi and A. Hmamed, "Improved finite frequency $H_{\infty}$ filtering for TakagiSugeno fuzzy systems," International Journal of Systems, Control and Communications, vol. 11, no. 1, pp. 1-24, 2020.

[26] A. El-Amrani, A. E. Hajjaji, I. Boumhidi and A. Hmamed, "Finite frequency $H_{\infty}$ filter design for TS fuzzy continuous systems," International Journal of System of Systems Engineering, vol. 10, no. 4, pp. 367-396, 2020.

[27] Tuan, H. D., Apkarian, P., Narikiyo, T., and Y. Yamamoto, "Parameterized linear matrix inequality techniques in fuzzy control system design," IEEE Transactions on fuzzy systems, vol. 9, no. 2, pp. 324-332, 2001.

[28] M. J., Lacerda, R. C. L. F., Oliveira and P. L. D., Peres, "Robust $H_{2}$ and $H_{\infty}$ filter design for uncertain linear systems via LMIs and Polynomail matrices," Signal Processing, vol. 91, no. 5, pp. 1115-1122, 2011.

[29] T. Iwasaki and S. Hara, "Generalized KYP lemma: Unified frequency domain inequalities with design applications", IEEE Trans. Autom. Control, vol. 50, No. 1, pp. 41-59, 2005.

[30] S. K. Nguang and W. Assawinchaichote, " $H_{\infty}$ filtering for fuzzy dynamical systems with D-stability constraints," IEEE Trans. Circuits Syst. I, Reg. Papers, vol. 50, no. 11, pp. 1503-1508, Nov. 2003. 